\title{
ForMation OF LARge SACLE STRUCTURES IN THE UNIVERSE BY INVERSE CASCADE: COSMOSYNERGETICS
}

\author{
V. Krishan and $C$. Sivaram \\ Indian Institute of Astrophysics, Bangalore - 560034, India
}

\begin{abstract}
It is suggested that inverse cascade that may occur in the turbulent cosmic medium can result in the formation of very large scale structures in the universe, upto the largest scales like the Great Wall. Again clustering of galaxies on all scales is interpreted to be due to these self-organisation processes occurring in a turbulent medium, the largest structures being anisotropic and nearly two dimensional, the smaller structures remaining isotropic. The observed fractal distribution of galaxies is also interpreted on this basis. The direct proportionality between the flow velocity and the 1 inear dimension of the structure may show a way out of the dil emma of missing matter.
\end{abstract}

\section{INTRODUCT ION}

The origin of the observed hierarchy of large scale cosmic structures continues to be a challenging problem in conventional cosmology. The observed distribution of galaxies into very ordered large scale formations strongly suggests the existence of well defined physical processed giving $r$ ise to self organised structures. A recent survey by Geller and Huchra of thousands of galaxies in a relatively narrow strip of space shows that galaxies follow intricate networks of arcs and segments with huge rounded cells. The conventional scenarios in which scale invariant density fluctuations grew in scale and amplitude with the expansion of the universe cannot form such large structures on a thbble time scale. The peculiar velocities of many galaxies indicate that they are being drawn towards enormous concentrations of matter with 
masses $\sim 10^{27} \mathrm{M}_{\odot}$. As shown by (Burstein et al.,1986) galaxies between the ${ }^{\circ}$ ocal supercluster and the Hydra Centaurus supercluster share same direction of motion showing peculiar velocities of several hundred kilometres per second, revealing presence of enormously massive structures $\sim 10^{16} \mathrm{M}_{\odot}$ powerful enough to draw several clusters towards it. More recently the Great Wall of galaxies Huchra and Geller (1989) has been identified over scales $\sim 100 \mathrm{Mpc}$ !

General observational indications are that the smaller scale structures 1 ike the globular clusters (containing the oldest stellar populations) formed first. Recent observations show that galaxies already existed at redshifts near $\mathrm{Z}=4$ thus giving $\mathrm{r}$ ise to the problem of how the smaller scale structures interacted leading to the formation of large structures such as clusters and super clusters of galaxies. In the conventional warm or hot dark matter scenario (as also in the Zeldovich type pancake fragmentation) it appears that the largest scale structures formed first. Then one is faced with the problem of accounting for smaller scale structures even at $\mathrm{Z}=4$. The cold dark matter scenario has had some measure of success in forming the smaller structures first but its essential ingredient is a particle (i. e. the axion) evidence for which is becoming more and more meagre. The rejuvenation of cosmic turbulence in galaxy formation through energy cascading from larger to smaller scales has been due to Ozernoi and coworkers $(1968,1971,1972)$. Cascading occurs for scales for which the hydrodynamic interaction time is smaller than cosmic expansion $t$ ime. For an expanding fluid with expansion rate given by the Friedmann equation the minimum scale over which dissipation can occur $l_{\min } \sim(\mathrm{Vt})^{\frac{1}{2}}$, obtained by equating time scales for dissipa-

tion (V) and expansion while the maximum length scale is given by maximum velocity of largest eddy, i.e.l $\max \sim v_{\max } t$; $v_{\max } \ll V_{s^{\prime}} V_{s}$ is the sonic speed. As a consequence of large Reynolds number and short hydrodynamic times, motions on comoving scales involving $l_{\min }<\ell<l_{\max }$ should rapidly acquire the Kolmogorov spectrum characteristic of ordinary turbulence in an incompressible viscous fluid. In an isolated turbulent medium, decay of large eddies into smaller eddies is a common occurrence. The conver se, i.e. small eddies coalescing into large ones can happen in an energetically open system, i.e. so called inverse cascading where the physical mechanism may be fusion of small-scale vortices into largerscale coherent structures, i.e. cluster-vortices. The cluster-vortices may be formed by an aggregation of "elementary" vortices (i.e. vortices with no cluster structure) to the cluster. Clusters of smaller sizes can in turn form through the aggregation of even smaller clusters. Such vortex clusters have been repeatedly observed in natural and numerical experiments. So here we suggest a definite physical mechanism for the large scale structure formation based on inverse 
cascading which quite naturally yields a bottom-up self similar hierarchical structure. In this picture, large hel ic ity fluctuations present in a turbulent medium play an important role in inverse cascading process. The helicity is defined as $\gamma=\vec{V} \cdot(\vec{\nabla} \times \vec{V})$. For a $3-D$ hydrod ynamic system in addition to total energy, there is a nother invariant $I$ which is defined as $I=C \int[E(K)]^{2} d K$ for a quasi-normal distribution of helicities. Using Kolmogorovic arguments, one $f i$ nd $\mathrm{S} E(\mathrm{~K}) \mathrm{k} \mathrm{K}-5 / 3$ and $\mathrm{EaL} \mathrm{L}^{2} / 3$ from energy invariance and from $I$ invariance, $E(K) K^{-1}$ and $E \alpha \log K(t) / l$ where $L(t)$ is the largest length scale excited at $t$ ime $t$. Lkvich and Tzvetkov $(1985)$, $K r$ ishan $(1980 \mathrm{a}, \mathrm{b})$, Kr1shan and Mogilevskii (1990) and Eruckner et al. (1999). Analogous to $2-D$ case (Hega 1985) one expects that in $3-D$ the invariant ' I' nould cascade towards large spatial scales and energy towards small spatial sales. The cascading of ' $I$ ' towards large spatial scales essentially enhances the correlation 1 ength of helicity fluctuations. We propose that this regime of turbulence favours formation of galaxy clusters at smaller scales. We can ask if there is an upper 1 imit to the size of these three-dimensional isotropic galaxy clusters (the lower 1 imit being determined by dissipation). A turbulent medium is restricted in the vertical direction by gravity. The largest dimension of fully $3-D$ structures is given by the ratio $I / E^{2}=L=L$ where $L$ is the characteristic vertical sale. Here we identify it with the size of clusters of galaxies $=0.3 \mathrm{Mpc}$. When the correlation length of helicity fluctuat ions reaches the 1 imit $L_{z}$, it can only grow in the horizontal plane, so that the system becomes more and more anisotropic. Thus the vertical component $V_{z}$ becomes independent of $(x, y, z)$ and the horizontal components $V_{x}, V_{y}$ independent of $Z$, the invariant $I$ becomes $I a V_{k}^{2}=K E(k) \alpha L^{2 / 3}$, or $I(k) a k^{-5 / 3}$ where $I$ is the largest length scale in the horizontal plane. Thus $I(K)$ spectrum coincides with energy spectrum of 2-D turbulence corresponding to inverse cascade. One expects an increasing fraction of energy to be transferred to large spatial scales as anisotropy increases and we consider this part of the turbulence spectrum conducive to formation of superclusters appearing predominantly on large horizontal scales with above energy spectrum. The intermediate region where the 3-D system is developing anisotropy the energy spectrum going as $E(K) a K^{-1}$, can be identified with the gap between scales of clusters of galaxies and superclusters. The growth of large structures in a highly anisotropic turbulence can be interrupted by symmetry breaking caused by the cor io$l$ is force, the length scale $L_{c}$ where the nonlinear NavierStokes term becomes comparable with the coriolis force is $\mathrm{L}_{\mathrm{C}} \sim \mathrm{V} / \Omega$, where $\Omega$ is the angular velocity. Given sufficient energy, structures of size $L_{c}$ must form. At these large spatial scales, the system simulates 2-D turbulence which conserves entrophy and energy. The enstrophy then cascades towards small scales with a power law spectrum given by $E(K) \alpha K^{-3}$ and $\mathrm{E} \alpha \mathrm{L}^{2}$. There is a break in the spectrum as energy must cascade 
to large spatial scales as $L^{2 / 3}$ and to small scales as $L^{2}$. If we take $L_{c}$ '. $L_{G C G}$ (size of giant clusters $\sim 100 \mathrm{Mpc}$ ) and a random velocity of superclusters $\sim 10^{4} \mathrm{~km} / \mathrm{s}$ one finds an angular velocity $\sim 3 \times 10^{-18} \mathrm{rad} / \mathrm{sec}$. If the energy of the larger structures has been inverse cascaded from smaller structures, the energy density in the large scale should not exceed that in the small scale. From the energy spectrum Ea $\mathrm{L}^{2}$, energy balance gives then:

$$
\mathrm{E}_{\mathrm{SCG}} \leq\left|\frac{\mathrm{E}\left(\mathrm{L}_{\mathrm{SCG}}\right)}{\mathrm{L}_{\mathrm{SCG}}^{2}{ }^{2}{ }_{\mathrm{GCG}}}\right|^{2 / 3} \mathrm{~L}_{\mathrm{GCG}} \leq \mathrm{E}\left(\mathrm{L}_{\mathrm{SCG}}\right)
$$

where []$^{2 / 3}$ is the enstrophy injection rate.

For $\mathrm{L}_{\mathrm{GCG}}=100 \mathrm{Mpc}, E\left(\mathrm{~L}_{\mathrm{SCG}}\right) \sim\left(10^{4}(\mathrm{~km} / \mathrm{s})^{2}\right.$ and $\mathrm{L}_{\mathrm{SCG}} \sim 3 \mathrm{Mpc}$, one gets $\tau_{G C G} \simeq 10^{11}$ years. So structures of the size of a

few hundred Mpc cannot be formed if the random velocity of a supercluster is less than $10^{4} \mathrm{~km} / \mathrm{s}$. For a supercluster

$\mathrm{L} \sim 3 \mathrm{Mpc}$ and $\mathrm{E}\left(\mathrm{L}_{\mathrm{CG}}\right)=(300 \mathrm{~km} / \mathrm{s})$, for random galactic cluster velocities we get $\tau \sim 3 \times 10^{9}$ years. The giant clusters are formed in a turbulent medium being stirred by random motion of superclusters. The eddy turnover time for the largest structure of say $100 \mathrm{Mpc}$ with the associated fluid velocity of $\sim 10^{4} \mathrm{~km} / \mathrm{s}$ is $\sim 10^{10} \mathrm{yrs}$, which is smaller than the tubble time. This is al so true for smaller scales. In this theory, the smaller structures are more or less isotropic whereas the larger ones are anisotropic and become nearly two dimensional at the largest scales forming giant sheets like the Great Wall. At the largest scales, VaL. This inbuilt 1 inear velocity-distance relation may account for flat rotation curves and velocities increasing with distance in giant clusters without the need to invoke unseen matter. Silk has shown that the effects of expansion causes 1 ittle deviation in turbulence from incompressible case. We can al so generalize the above results to fractal turbulence aga in considering an inverse cascade, i.e. clustering of vortices. These clusters join with one another into even larger clusters etc. (a hierarchical cascade). It is clear that the energy dissipated in a volume of characteristic size $r$ on vortices of size $r$ (roughly true for galaxies as galaxy size $\sim$ galaxy separation) must be proportional to number of elementary vortices in the fractal cluster vortex. Denoting this by $\mathrm{N}_{\mathrm{d}}$ and the dissipated power by $W_{d}$ then for the isotropic case we can write Monin and Yaglom (1975), Ber shadskii (1989):

$$
w_{d} \text { a } r^{d} \rho K^{2} E(K) d K
$$


where $d$ is the dimension of space in which the motion (here $d=3$ ). Assuming that the scale $r^{-1}$ belongs to the scaling range where $\mathrm{Ea} \mathrm{K}-\alpha$ is satisfied, we obtain

$$
W_{d} \propto r^{d+\alpha-3}
$$

For a Fractal cluster Snirnov (1976)

$$
N_{d} \propto e^{D}
$$

Thus $\alpha=D+3-\alpha$, so that for 3-dim-turbulence $\alpha=D$, i.e. scaling exponent in the spectral law $E^{\alpha} K^{-\alpha}$ for 3-dim isotropic turbulence is equal to the fractal dimension of the cluster vortex correlation. For the fractal dimension of the clusters formed by inverse cascade one may use the value given by Kolb (1984) for hierarchical clustering, This applies to the situation, where both direct cascading and clustering occur simultaneously, i.e. $D=1.72$, so that we obtain for three-dimenstonal isotropic turbulence, the fractal dimension of the correlation as $D=1.72$, which is in good agreement with the value of $\sim 1.8$ generally used empirically for galaxy correlation. Fractal dimension, according to current experiment data, for inver se cascade 1.69-1.72 Yaglom (1985). For the strictly Kolmogorov case in strictly three dimensions, $D=5 / 3=1.66$ (as argued above $\alpha=D$ in the $E^{-\alpha}$ relation)not far from the above value. This shows that Kolmogorov Scaling can be valid for hierarchical galaxy cluster formation. In short we have for the $f$ irst time the picture of inverse cascading process (with its many attractive features) for formation of a whole hierarchy of large sale cosmic structures.

\section{NOTE ON INFLATION}

In the above picture we did not take into account, the earliest epochs of the universe, which according to popular thinking is an exponential expansion of the scale factor as $R=R_{0} \exp (H t)$. It has been argued that such an exponential expansion would considerably dilute all initial vorticity and magnetic $f i e l d s$ if any. For instance with flux conservation assumed an initial magnetic field would be diluted by a factor of $\exp (2 \mathrm{Ht}) \sim e^{60}$ for most injection models. Hwever, this need not necessarily be so. There are very recent models where B decreasing as $1 / \mathrm{r}^{2}$ is averted by breaking conformal invariance of electromagnetism by considering non-minimal gravitational couplings of photon field to gravity such as $R^{2}, R F^{\mu v}$ $F^{H V}$ etc. These terms in fact results in production of large scale magnetic fields during inflation, Turner and Widrow (1988) al so De Sabbata and Sivaram (1988), for post inflation generation of magnetic field through vorticity in general relativity. Since magnetic fields can be coupled to differential rotation of ions and electrons in the plasma prior to recombination. 
This would also generate vorticity in addition to magnetic field. Moreover MHD turbulence aids inverse cascading. So inflationary expansion in the earliest phase need not be a drawback to this picture which gives a scale-invariant description independent of inflation. Since we have only acoustical dissipation of energy (viscosity), it would not couple to the photons of the microwave background (i.e. no thermal effects). So no large scale distortion of the microwave background is expected in this process. (The amount of vorticity needed on the largest scales is consistent with the anisotropy of the microwave background (since we are just translating large scale peculiar velocities with turbulent velocities).

\section{REFERENCES}

[1] Bershadskii, A.G.(1989), Sov.Phys. JETP, 69, 354 .

[2] Bruckner, D., Cook, J. and Krishan, V. (1989), in "Solar Inter ior and Atmo sphere" Univ. of Arizona Press Ed: W.Livingston et al.

[3] Burstein, D. et al. (1986), in "Galaxy Distances and Deviations from Universal Expansion" $\mathrm{Ed}$ : B.Madove and R.B. Tul1y, p.123.

[4] DeSabbata and Sivaram, C., (1988), Nuovo Ci, 102 B, 107 .

[5] Hasegawa, A. (1985), Advances in Physics, 34,1 .

[6] Huchra, J. and Geller, M. (1989), Science, 246,897.

[7] Krishan, V. (1990a), Proceedings of IAU Symposium 138, Ed.J. Stenflo.

[8] Krishan, V. (i990b), Mon. Not. Roy. Astron.Soc. (To appear).

[9] Krishan, V. and Mogilevskij, E.I. (1990), Proceedings of IAU Symposium 142, Ed. E.R. Priest and V.Krishan.

[10] Kol b, M. (1984), Phys. Rev. Letts. 53,1653.

[11] Levich, E. and Tzvetkov, E. (1985), Phys. Reports $128,1$.

[12] Monin,A.S. and Yaglom, A.M.(1975), Statistical Hydrodynamics, M.I.T., Press Cambridge.

[13] Ozernoi, L.M. and Charnin, A.D. (1968), Sov. Astron.A.J. 12,901 .

[14] Ozernoi, L.M. and Chibisov, G.V. (1971), Astrophys.Lett. 7, 201.

[15] Ozernoi, L.M. and Chibisov, G.V. (1972), Sov. Astron.A.J. 15,923 .

[16] Smirnov, B.M. (1986), Sov. Phys. Uspekhi, $29,481$.

[17] Turner, M.S. and Widrow, L.M. (1988), Phys. Rev. D37, 2743.

[18] Yaglom, A.M. (1985) in "A.N. Kolmogorov", Selected papers Nauka Moscow. 